\title{
FORMACIÓN AUTONÓMICA EN LA INTERRUPCIÓN DEL EMBARAZO
}

\section{Cruz García-Lirios 1}

Universidad Autónoma Metropolitana, México

Resumen.-El objetivo del presente trabajo es discutir ta relación entre la autonomía como resultado de una formación sanitaria en torno a la solicitud de interrupción legal del embarazo, consíderando los marcos teóricos, conceptuales y empíricos revisados durante el periodo que va de 2000 a 2018, su indexación en repositorios internacionales como Dialnet, Latindex, Redalyc y Scielo, así como su búsqueda, selección y procesamiento a partir de las categorías de sexualidad, autonomía e interrupción legal del embarazo. Se proponen líneas de investigación acordes a la literatura consultada.

Palabras clave: Sexualidad, Autonomía, Embarazo, Formación, Educación

\section{Autonomic training in the legal interruption of pregnancy}

Abstract.- The objective of this work is to discuss the relationship between autonomy as a result of health training around the request for legal interruption of pregnancy, considering the theoretical, conceptual and empirical frameworks reviewed during the period from 2000 to 2018, its indexation in international repositories such as Dialnet, Latindex, Redalyc and Scielo, as well as its search, selection and processing based on the categories of sexuality, autonomy and legal interruption of pregnancy. Research lines are proposed according to the literature consulted.

Keywords: Sexuality, Autonomy, Pregnancy, Training, Education

\section{Introducción}

El objetivo del presente trabajo es establecer los ejes y dimensiones del sistema familiar en relación con la formación de la autonomía de la mujer con respecto a sus derechos humanos en general y su capacidad de elección en particular frente a problemáticas de salud reproductiva y sexual que afectan su desarrollo.

En el marco de los derechos universales, las mujeres han sido un sector vulnerado en cuanto a la violación de sus privilegios y capacidades de

\footnotetext{
1 Universidad Autónoma Metropolitana: csh96327267@titlani.uam.mx
} 
elección. Se estima que, en América Latina, una mujer tendrá hasta cinco veces más probabilidades de ser agredida con violencia con respecto a otras regiones (García, Carreón, Hernández, Montero y Bustos, 2012).

En ese tenor de violencia contra la integridad de la mujer, los delitos calificados de odio contra el género femenino y las políticas de derechos humanos han centrado su interés en la formación de la autonomía y la composición de las familias como escenario de empoderamiento y defensa de los derechos de la mujer (Bautista, Delgado, García, Valdés, Hernández, Castro y Trujillo, 2016).

En tal sentido es que la familia ha sido observada como un sistema en el que interactúan estilos de vida libres de violencia con funciones y estructuras de equidad e inclusión en la toma de decisiones frente a contingencias o riesgos asociados a la salud de la mujer como sería la interrupción legalmente asistida del embarazo (García, Hernández, Aguilar y Morales, 2016).

Más específicamente, los estilos de vida familiares libres de violencia generan emociones, creencias y objetivos centrados en el desencadenamiento de la equidad, la confianza y la responsabilidad. Además, las familias inciden en las decisiones de la mujer propiciando un incremento y reducción de la motivación para llevar a cabo una acción de autocuidado o preventiva como sería la solicitud de interrupción del embarazo (García, Hernández, Morales, Aguilar, García y Hernández, 2015).

Es así como la autonomía, en tanto proceso formativo sugiere la inclusión de factores en seno familiar que permiten no sólo la protección de los derechos de la mujer sino además su desarrollo con base en la toma de decisión que más convenga a sus intereses (Carreón, Bustos, Hernández, Quintero y García, 2015).

En tal escenario, la familia es un factor que regula la entrada y la salida de emociones, información y eventos que favorecen la vida libre de violencia. Se trata de un catalizador de datos que procesa en función de la importancia que tiene la mujer en la estructura de una familia, pero sobre todo a partir de los aportes de la mujer a la dinámica familiar (García, Morales, Méndez, Delgado, Vilchis y López, 2015).

En el caso de la interrupción asistida del embarazo el principal factor que determina la solicitud es el tipo de relación con la pareja, pero cuando la mujer percibe que su relación sentimental es poco formal, entonces la influencia de la madre es fundamental al momento de decidir la solicitud de aborto (Delgado, Méndez, Morales, García, Mendoza y Vilchis, 2018).

De este modo, la autonomía femenina resignifica no sólo la equidad y la responsabilidad sino además la composición de la familia y su influencia 
en el desarrollo de ambientes libres de violencia y autocuidado (García, Carreón, Hernández, Bustos, Morales y Limón, 2013).

Cabe destacar que, en el proceso de formación autonómica de la mujer, el empoderamiento ha sido catalogado como un factor esencial para el establecimiento de las relaciones y ambientes libres de violencia y factibles al desarrollo femenino, pero supone caminos distintos ante los eventos de riesgo como la interrupción asistida del embarazo (García, Carreón, Hernández, Bustos, Bautista, Méndez y Morales, 2013).

En ese tenor, la formación autonómica femenina supone fases y metas que van desde la misión y la visión de los talentos hasta el incremento de la autovaloración, pero también puede reorientarse hacia el replanteamiento de objetivos, tareas y metas (García, Morales, Méndez, Delgado, Vilchis y López, 2016).

Ahora bien, la importancia de la autonomía no radica en que es la culminación de un sistema social para el bienestar personal de quien toma sus decisiones con base en la equidad y la responsabilidad, sino más bien, radica en los efectos que la autonomía genera en las motivaciones e intenciones de los sujetos proactivos (Rincón, López y García, 2018).

A menudo, la autonomía al interactuar con otros factores como las habilidades y los propósitos genera la motivación que impulsará los proyectos individuales y familiares, resaltando la función catalizadora de la familia y la importancia del desarrollo de las capacidades personales (Sandoval, García y Bustos, 2016).

En efecto, la autonomía es parte de un ciclo de libertad y emprendimiento que tendría su reflejo principal en el establecimiento de la transformación de la personalidad individual o la conformación de un grupo (García, 2007a; 2007b).

En el caso de la interrupción asistida del embarazo, la autonomía es parte de un proceso en el que el evento traumático y postraumático se reduce a su mínima expresión gracias al procesamiento del evento en el que la autonomía es un catalizador esencial y que, con otros factores que se generan en el seno familiar potencializa las posibilidades de éxito de quienes poseen un sentido de pertenencia al lugar, el grupo y la situación.

En síntesis, la formación autonómica femenina consiste en el establecimiento de un ciclo de aversión a los riesgos y propensión a estilos de vida libres de violencia. Se trata de un proceso en el que la autonomía juega un papel crucial en la consecución de objetivos, el establecimiento de tareas y el logro de metas (García, 2008).

\section{Teoría de la formación sexual}


Los marcos teóricos y conceptuales que explican la formación sexual son: 1) Teoría del Interculturalismo Sexual, 2) Teoría de la Identidad de Género y 3) Teoría de la Conducta Planificada (véase Figura 1).

Figura 1. Teoría de la Formación Sexual

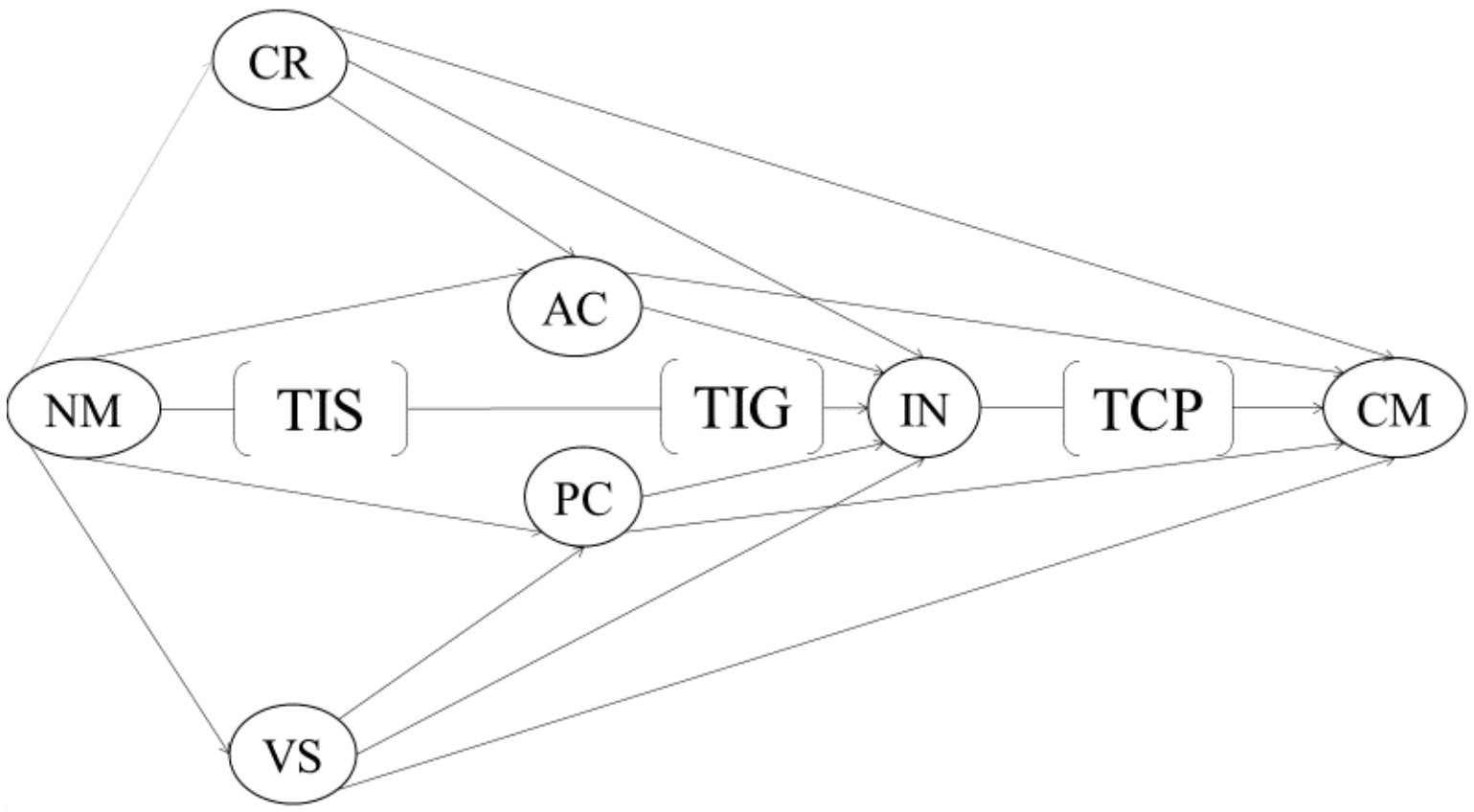

TIS = Teoría del Interculturalismo Sexual, TIG = Teoría de la Identidad de Género, TCP = Teoría del Comportamiento Planificado: NM = Normas, VS $=$ Valores, $\mathrm{CR}=$ Creencias, $\mathrm{AC}=$ Actitud, $\mathrm{PC}=$ Percepción, $\mathrm{IN}=$ Intención, $\mathrm{CM}=$ Comportamiento

Fuente: Elaboración propia

La autonomía, entendida como criterios derivados de la interrelación entre diversas culturas, es identificada como una norma subjetiva con valores de colaboración y disposiciones favorables hacia los derecho sexuales y reproductivos (García, 2011).

En tal sentido, la teoría del interculturalismo considera a la autonomía como un factor que antecede a la toma de decisiones informada y sobre todo respaldada por representantes de minorías activas que demandan el servicio público. Se trata de una instancia jurídica indispensable para la protección social de grupos vulnerables como es el caso de las adolescentes que viven en situación de embarazo no deseado (García, 2012a: 2012b; 2012c).

Sin embargo, el interculturalismo sexual al poner énfasis sobre la representación de las minorías más que de la promoción de los derechos sexuales orienta la discusión hacia la identidad de género como motor de difusión intercultural y diversidad sexual. Es así como la teoría de la identidad de género sostiene que la masculinidad hetero patriarcal dominante es el factor que inhibe no sólo el ejercicio de los 
derechos sexuales y reproductivos sino, además la discusión sobre la diversidad sexual (García, 2007a).

En entornos laborales, la teoría de la identidad de género edificó protocolos de difusión de los derechos sexuales y reproductivos en el marco de la salud ocupacional, pero la práctica de la solicitud de interrupción del embarazo no fue posible hasta que la teoría del comportamiento planificado delimitó la toma de decisiones a partir del control percibido de la información (García, Valdés, Sánchez, Elizarraráz, Méndez y Hernández, 2015).

La teoría del comportamiento planificado, a diferencia del interculturalismo jurídico y la teoría de la identidad de género promotora de derechos sexuales y reproductivos, sugiere que la solicitud de interrupción del embrazo no debiera darse en una situación apremiante y más bien debiera ser resultado de una planificación familiar (García, 2012a).

Sin embargo, los estudios de la formación sexual advierten que incluso aquellos que han asistido a cursos de sexualidad, asertividad y uso del condón engrosan las listas de los embarazos no deseados y las solicitudes de interrupción legal del embarazo (García, 2007b).

\section{Estudios de la formación sexual}

La Tabla 1 muestra los estudios de la formación sexual entre los que destacan; a) elección de pareja sexual, b) uso de condón, c) interrupción del embarazo.

Tabla 1. Estudios de la formación sexual

\begin{tabular}{cll}
\hline Año Autor & Resultados \\
\hline 2010 & Fernández & Encontraron que el rango de edad en el que más se \\
& practican abortos es del 19 por ciento para la \\
muestra de entre 26 y 30 años. El 16 por ciento se ve & obligado a abortar por la presión de la pareja, \\
familia o su situación económica baja.
\end{tabular}

2010 Oduwole A medida que el compromiso con su pareja se prolonga, la elección de pareja es más evidente sobre las intenciones impersonales o unilaterales

2011 Serrano Demostró un incremento de los conocimientos sobre la prescripción abortiva de emergencia en relación con los años de residencia médica. En la medida en que el tiempo de residencia se incrementaba, se observaba un mayor porcentaje de conocimientos sobre la prescripción abortiva de emergencia.

2011 Olaitan Las diferencias existentes entre hombres y mujeres respecto al sexo consensuado, anticoncepción y aborto inducido suponen al uso de dispositivos como 
instrumento de anticoncepción si las relaciones son ocasionales e infrecuentes

2011 Petracci En los casos opuestos, el coito interruptus es la práctica sexual más prevaleciente en hombres con un compromiso afectivo significativo

2011 Serrano Hombres y mujeres evalúan maternal 0 paternalmente a su pareja según sea el caso. Una evaluación positiva implica decisiones compartidas. En cambio, una autoevaluación negativa determina una decisión delegada a la pareja

2011 Lanre Establecieron diferencias entre las actitudes hacia el aborto con respecto a la condición de la madre en referencia al bebe. La legalización fue considerada como una medida de prevención ante el incremento de la práctica abortiva clandestina.

2011 Serrano La anticoncepción de emergencia fue el instrumento idóneo para prevenir embarazos no deseados, la frecuencia de prescripción de la píldora aumentó el $20 \%$,

2013 Cosmas Los efectos secundarios fueron considerados como el principal efecto secundaría de las píldoras contraceptivas. Las mujeres al seguir el consejo matriarcal de contracepción pueden ser inducidas a utilizar otros métodos tradicionales y modernos.

2014 García et Llevaron a cabo una discusión sistemática de los al., factores determinantes de la elección de pareja y la decisión de interrupción del embarazo, encontrando que de acuerdo al tipo de relación informal o formal las parejas delegan o acuerdan la interrupción asistida.

2015 García et Revisaron la literatura correspondiente de 2000 a al., 2018 para especificar un modelo con el fin de estudiar la relación entre la elección de pareja y la interrupción del embarazo, establecieron las trayectorias de relaciones de dependencia entre las variables para anticipar los efectos de una decisión unilateral respecto a un consenso en la pareja.

2016 Bautista et Establecieron la confiabilidad y la validez de un al., instrumento que midió la elección de pareja y la interrupción del embarazo a partir de variables socioculturales como los valores y las normas, variables sociodemográficas como el sexo, la edad, la escolaridad, el ingreso y el estado civil, así como 
variables sociocognitivas como las percepciones, actitudes e intenciones, encontrando que su interrelación determina indirectamente la solicitud de interrupción del embarazo.

2017 García et Contrastaron un modelo estructural de trayectorias al., híbridas entre los factores determinantes de la elección de pareja y la interrupción del embrazo, encontrando que la ruta de percepciones de control, intenciones y comportamiento explican con el mayor porcentaje de varianza al fenómeno en comento.

2018 Delgado etal.,
Interpretaron los discursos de parejas en torno a la interrupción del embarazo con la finalidad de develar el significado del aborto, el cual fue construido desde una aversión a los costos más que desde una propensión a los beneficios.

Fuente: Elaboración propia

Los estudios muestran la prevalencia de la elección de pareja como determinante de la toma de decisiones en torno a la interrupción legalmente asistida del embarazo. De esta manera, el tipo de relación de pareja es determinante de la toma de decisiones consensada, 0 bien, el delegamiento unilateral a la pareja (García, 2016).

Sin embargo, otros estudios advierten que la interrupción legal del embarazo es resultado de una difusión intensiva de los derechos sexuales y reproductivos, así como de la salud ocupacional en el ámbito laboral como serían los casos de permisos por maternidad o paternidad, así como la demanda de guarderías en los espacios laborales (García, Carreón y Hernández, 2014).

Los estudios de la interrupción legal del embarazo también centran su atención en las diferencias socioeconómicas de sectores vulnerados al embarazo no deseado como serían los casos de centros marginales, barrios populares y zonas residenciales de clase media (García, 2018).

Se advierte la prevalencia de la virilidad como símbolo de control y dominio de los hombres hacia las mujeres en desarrollo marginal, la galanura o galantería como símbolo de relación de pareja en las clases populares y la opulencia como símbolo de estatus económico en las parejas de clases medias (García, 2012b).

\section{Método}

Se realizó un estudio documental con una selección intencional de fuentes indexadas a repositorios internacionales como Dualnet, 
Latindex, Redalyc y Scielo, considerando el periodo que va de 2000 a 2018, así como las palabras claves de sexualidad, autonomía y formación (véase Tabla 2).

Tabla 2. Descriptivos de la muestra informativa

\begin{tabular}{cccc}
\hline & Sexualidad & Autonomía & Formación \\
\hline Dialnet & 28 & 17 & 10 \\
\hline Latindex & 16 & 9 & 7 \\
\hline Redalyc & 9 & 3 & 5 \\
\hline Scielo & 2 & 1 & 1 \\
\hline
\end{tabular}

Fuente: Elaborada con los datos del estudio

Se construyó una matriz de análisis de contenido, siguiendo la técnica Delphi la cual sugiere la comparación de datos a partir de su diferenciación contextual y sintética, así como la integración de información a partir de sus similitudes advertidas por jueces que evaluaron los datos (véase Tabla 3).

Tabla 3. Construcción de la matriz de análisis de contenido

\begin{tabular}{|c|c|c|c|c|}
\hline & Concepto & Indicador & Medición & Interpretación \\
\hline Sexualidad & $\begin{array}{l}\text { Refiere a la } \\
\text { expresión } \\
\text { afectiva, } \\
\text { emocional o } \\
\text { sentimental de } \\
\text { personas en } \\
\text { situación de } \\
\text { embarazo no } \\
\text { deseado } \\
\text { (García, } \\
\text { 2007a) }\end{array}$ & $\begin{array}{l}\text { Datos relativos } \\
\text { a la tasa de } \\
\text { embarazos, } \\
\text { maternidad, } \\
\text { abortos } \\
\text { defunciones y }\end{array}$ & $\begin{array}{l}\text {-1 para } \\
\text { información } \\
\text { negativa, } 0 \\
\text { para } \\
\text { información } \\
\text { desvinculada } \\
\text { y + } 1 \text { para } \\
\text { información } \\
\text { positiva }\end{array}$ & $\begin{array}{l}\text { Altos puntajes } \\
\text { corresponden } \\
\text { a una } \\
\text { sexualidad } \\
\text { desarrollada }\end{array}$ \\
\hline Autonomía & $\begin{array}{l}\text { Refiere a toma } \\
\text { de decisiones } \\
\text { informadas } \\
\text { con respecto } \\
\text { a los derecho } \\
\text { sexuales y } \\
\text { reproductivos } \\
\text { (García, } \\
\text { 2007b) }\end{array}$ & $\begin{array}{l}\text { Datos alusivos a } \\
\text { solicitudes de } \\
\text { interrupción } \\
\text { legalmente } \\
\text { asistida del } \\
\text { embazo } \\
\text { unipersonales y } \\
\text { en pareja }\end{array}$ & $\begin{array}{l}\text {-1 para } \\
\text { información } \\
\text { negativa, } 0 \\
\text { para } \\
\text { información } \\
\text { desvinculada } \\
\text { y + } 1 \text { para } \\
\text { información } \\
\text { positiva }\end{array}$ & $\begin{array}{l}\text { Altos puntajes } \\
\text { aluden a una } \\
\text { autonomía } \\
\text { planificada y } \\
\text { orientada a } \\
\text { la salud } \\
\text { sexual y } \\
\text { reproductiva }\end{array}$ \\
\hline Formación & $\begin{array}{l}\text { Refiere al } \\
\text { procesamiento } \\
\text { de }\end{array}$ & $\begin{array}{l}\text { Datos relativos } \\
\text { a } \\
\text { conocimientos }\end{array}$ & $\begin{array}{l}-1 \quad \text { para } \\
\text { información } \\
\text { negativa, } 0\end{array}$ & $\begin{array}{l}\text { Altos puntajes } \\
\text { corresponden } \\
\text { a educación }\end{array}$ \\
\hline
\end{tabular}




\begin{tabular}{|c|c|c|c|}
\hline $\begin{array}{l}\text { información } \\
\text { concerniente } \\
\text { a la salud } \\
\text { sexual y } \\
\text { reproductiva } \\
\text { (García, } \\
\text { 2007c) }\end{array}$ & $\begin{array}{l}\text { y habilidades } \\
\text { de } \\
\text { anticoncepción }\end{array}$ & $\begin{array}{l}\text { para } \\
\text { información } \\
\text { desvinculada } \\
\text { y + } 1 \text { para } \\
\text { información } \\
\text { positiva }\end{array}$ & $\begin{array}{l}\text { para la } \\
\text { sexualidad y } \\
\text { la } \\
\text { reproducción } \\
\text { planificadas }\end{array}$ \\
\hline
\end{tabular}

Fuente: Elaboración propia

La información se procesó en el paquete de análisis cualitativo de datos (QDA por su acrónimo en inglés versión 4,0).

\section{Resultados}

La Figura 3 muestra la propuesta de estudio de las trayectorias de relaciones de dependencia entre los factores revisados o inferidos de la literatura.

Figura 3. Modelo para el estudio de la autonomía en la interrupción del embarazo



VEP $=$ Valores en la Elección de Pareja, CEP = Creencias en la Elección de Pareja, PRE = Percepciones en la Relación de Pareja, AIE = Actitudes hacia la Interrupción del Embarazo, IIE = Intención de Interrupción del Embarazo, SIE = Solicitud de Interrupción del Embarazo

Fuente: Elaboración propia

Es posible observar cinco hipótesis de los escenarios y las situaciones en torno a las trayectorias de relaciones de las variables explicativas de la solicitud de interrupción del embarazo que, pueden ser resultado de la 
interrelación de valores, creencias, percepciones, actitudes e intenciones (hipótesis 1).

O bien, la solicitud no se establece, pero la intencionalidad prevalece como resultado de las cadenas de sucesos que van de los valores, creencias, actitudes e intenciones (hipótesis 2), aunque también puede ser la interrupción del embarazo una disposición desfavorable 0 favorable que se explica desde las percepciones, creencias y valores (hipótesis 3).

Sin embargo, la cuestión relativa a la interrupción del embarazo puede nos ser un tema central en la agenda de las parejas y en el mejor de los casos un asunto latente que estaría detrás de la percepción del tipo de relación de pareja (hipótesis 4) y de las creencias de elección de pareja (hipótesis 5).

\section{Consideraciones finales}

El aporte del presente trabajo al estado de la cuestión fue la especificación de modelo para el estudio de la autonomía en la solicitud de interrupción del embarazo, aunque el tipo de estudio documental, el tipo de muestreo intencional y el tipo de análisis de contenido limitan los resultados al contexto de la investigación, sugiriendo el uso del modelo en escenarios planificados de elección de pareja.

Delgado et al., (2018), García (2018) y Rincón, López y García (2018) llevaron a cabo revisiones, discusiones y especificaciones de modelos en torno a la sexualidad consensuada, el uso del condón y la solicitud de interrupción del embarazo para establecer el denominador común en la identidad de género, pero en el presente trabajo además se ha propuesto un modelo a partir del interculturalismo y la planificación.

Es menester llevar a cabo especificación y pruebas de modelos con la finalidad de establecer los contextos de formación de la sexualidad que anticipen las elecciones de pareja orientadas al embarazo no deseado y la solicitud de interrupción del embarazo como instrumento de ausencia de planificación y consenso en las relaciones de pareja.

\section{Referencias}

Bautista, M., Delgado, M. A., García, C., Valdés, O., Hernández, J., Castro, A. y Trujillo, K. V. (2016). Contrastación de un modelo de cultura organizacional en centros de salud comunitaria ante la difusión informativa del VIH / SIDA desde el Trabajo Social. Enseñanza e Investigación en Psicología, 21 (3), 248-255

Carreón, J., Bustos, J. M., Hernández, J., Quintero, M. L. y García, C. (2015). Confiabilidad y validez de un instrumento que mide la actitud hacia grupos cercanos a portadores del VIH / SIDA. Eureka, 12 (2), 218230 
Delgado, M. A., Méndez, A., Morales, M. L., García, C., Mendoza, D. y Vilchis, F. J. (2018). Contraste de un modelo de disposición a la solicitud del embarazo. Eurytmie, 5, 1-12

García, C. (2007a). La percepción de utilidad del comercio electrónico. Enseñanza e Investigación en psicología, 12 (2), 409-420

García, C. (2007b). Una teoría del consumo móvil. Sociedad Hoy, 13, 924

García, C. (2008). La brecha digitat- de las generaciones futuras. Entelequia, 8, 61-72

García, C. (2011). Actitudes hacia las relaciones destructivas en el noviazgo. Revista de Psicología, 15 (38), 1-8

García, C. (2012a). Actitudes hacia el aborto legal asistido. Documentos de Trabajo Social, 50, 269-279

García, C. (2012b). Estructura de la intención asertiva en el noviazgo. Poiesis, 23, 1-9

García, C. (2012c). Estudio de las actitudes hacia el aborto en universitarios de Morelos, México. Xhimai, 7 (13), 61-82

García, C. (2014). Uso de internet en un sistema público educativo. Educación y Futuro Digital, 10, 65-80

García, C. (2016). Emprendimiento digital: Estudio de caso en universitarios de comunicación, UAEM-UAP Huehuetoca. Revista de Ciencias Sociales, 29, 34-45

García, C. (2018). Redes informativas de salud comunitaria en una localidad del centro de México. Epsys, 12, 1-20

García, C., Carreón, J. y Hernández, J. (2014). Campos discursivos sexistas: Estado del conocimiento. Margen, 73, 1-15

García, C., Carreón, J., Hernández, J., Bustos, J. M., Bautista, M., Méndez, A. y Morales, M. L. (2013). Confiabilidad y validez de un instrumento de medición en búsqueda de Internet. Revista de Psicología de la Universidad de Antioquía, 5 (1), 27-34

García, C., Carreón, J., Hernández, J., Bustos, J. M., Morales, M. L. y Limón, G. A. (2013). Dimensione utilitarista y eficientista en el uso de Internet. Visión gerencial, 12 (2), 281-292

García, C., Carreón, J., Hernández, J., Montero, M. y Bustos, J. M. (2012). Confiabilidad y validez de un instrumento que mide la percepción de eficiencia de uso de Internet en una biblioteca pública de México, Distrito Federal. Invurnus, 7 (2), 3-11

García, C., Hernández, J., Aguilar, J. A. y Morales, M. L. (2016). Confiabilidad y validez de un instrumento que mide determinantes perceptuales del acoso a través de Internet. Encuentros, 14 (1), 103-117 
García, C., Hernández, J., Aguilar, J. A., Morales, M. L. y Peralta, M. V. (2016). Confiabilidad y validez de un instrumento que mide la intención de ciber-selectividad. Poiesis, 31, 6-18

García, C., Hernández, J., Morales, M. L., Aguilar, J. A., García, E. y Hernández, G. (2015). Contraste de un modelo de los indicadores psicológicos reflejantes de la eficiencia computacional. Compendium, 4, 31-43

García, C., Morales, M. L., Méndez, A., Delgado, M. A., Vilchis, F. J. y López, S. (2015). Confiabilidad y validez de un instrumento que mide percepciones de sexualidad. Rumbos, 10 (12), 87-93

García, C., Morales, M. L., Méndez, A., Delgado, M. A., Vilchis, F. J. y López, S. (2016). Estructura factorial exploratoria en torno a variables relativas a la interrupción del embarazo en estudiantes. Revista del Instituto de Salud Pública de la Universidad veracruzana, 12 (23), 15-28

García, C., Valdés, O., Sánchez, R., Elizarraráz, G., Méndez, A. y Hernández, J. (2015). Diferencias entre emprendedores internautas con respecto a la empatía, percepciones de riesgo y uso de aplicaciones tecnológicas. Perspectivas, 2 (1), 68-75

Organización de Naciones Unidas (2015). Desarrollo humano. New York: ONU

Rincón, R. M., López, S. y García, C. (2018). Contrastación de un modelo para el estudio de la intención de uso de Facebook en una universidad pública del centro de México. Margen 89, 1-8

Sandoval, F. R., García, C. y Bustos, J. M. (2016). Estructura factorial exploratoria del aborto planificado en estudiantes de Xochimilco, Ciudad de México. Prospectivas en Psicología, 2 (2), 40-50 